\title{
Potential application of hydrogen in traumatic and surgical brain injury, stroke and neonatal hypoxia-ischemia
}

\author{
Jan M Eckermann ${ }^{1,5^{*}}$, Paul R Krafft ${ }^{3}$, Lorelei Shoemaker ${ }^{1}$, Robert E Lieberson ${ }^{1}$, Steven D Chang ${ }^{1,2}$ and \\ Austin Colohan ${ }^{4^{*}}$
}

\begin{abstract}
This article summarized findings of current preclinical studies that implemented hydrogen administration, either in the gas or liquid form, as treatment application for neurological disorders including traumatic brain injury (TBI), surgically induced brain injury (SBI), stroke, and neonatal hypoxic-ischemic brain insult (HI). Most reviewed studies demonstrated neuroprotective effects of hydrogen administration. Even though anti-oxidative potentials have been reported in several studies, further neuroprotective mechanisms of hydrogen therapy remain to be elucidated. Hydrogen may serve as an adjunct treatment for neurological disorders.
\end{abstract}

Keywords: Hydrogen, Neuroprotection, Oxidative stress, Reactive oxygen species

\section{Introduction}

Traumatic brain injury (TBI) and cerebral vascular events can be devastating; yet there are few treatments proven to ameliorate the brain damage and overall outcome in patients. An ideal neuroprotectant would be non-toxic, easily administered, permeable at the bloodbrain barrier (BBB), and offer protection at all stages of injury, including prophylaxis. Hydrogen sulfide $\left(\mathrm{H}_{2} \mathrm{~S}\right)$ has shown some of these properties $[1,2]$. Its mechanisms may be related to the attenuation of reactive oxygen species (ROS) [3], or to its role as a neuromodulator [4]; however, the use of $\mathrm{H}_{2} \mathrm{~S}$ is controversial because of its toxicity [5] and gasotransmitter functions [6].

Hydrogen is the most abundant element in the universe [7]. Room air concentrations of hydrogen gas higher than $4 \%$ (normal air content $=0.000055 \%$ ) are explosive, and could cause asphyxiation [8]. Therapeutically relevant dosages of hydrogen, as used in the following animal studies (range 2\%-2.9\%), appear to be well tolerated. Furthermore, 3\% hydrogen gas has been

\footnotetext{
* Correspondence: jmeckermann@yahoo.com; acolohan@llu.edu 'Department of Neurosurgery, Stanford University School of Medicine, Stanford, California, USA

${ }^{4}$ Department of Neurosurgery, Loma Linda University School of Medicine, Loma Linda, California, USA

Full list of author information is available at the end of the article
}

safely and regularly used for human deep-sea divers without any adverse events [9].

Currently, to our knowledge, there are no FDA approved therapeutic regimens involving hydrogen gas or dissolved hydrogen. We sought to analyze recent available data regarding the use of hydrogen as a neuroprotectant.

Hydrogen therapy for traumatic brain injury and cerebral vascular events

\section{Traumatic brain injury}

Trauma is the leading cause of death in Americans younger than 45 years of age, and traumatic brain injury (TBI) accounts for over $50 \%$ of this mortality [10]. Out of the 1.5 million Americans who sustain a TBI each year, 230,000 are hospitalized, 80,000 to 90,000 remain with long-term disabilities, 50,000 die, and the estimated annual cost exceeds $\$ 60$ billion [11-13]. To investigate whether hydrogen $\left(\mathrm{H}_{2}\right)$ exerts abilities to ameliorate the outcome after TBI, Ji et al. administered $2 \% \mathrm{H}_{2}$ to rats, which were also subjected to experimental TBI [14]. TBI-challenged rats, that did not inhale $\mathrm{H}_{2}$, presented with significantly greater brain edema, blood-brain barrier (BBB) permeability, lesion size, and neurological impairments, than those in the treatment group. Decreased levels of oxidative products such as 
malondialdehyde (MDA) and 8-iso-prostaglandin F2 $\alpha$ (8-iso-PF2 $\alpha$ ), were decreased in brain tissues of treated animals, suggesting that $\mathrm{H}_{2}$ induces neuroprotection by reducing oxidative stress to the $\mathrm{BBB}$.

\section{Surgical brain injury}

Each year, approximately 800,000 patients undergo neurosurgical procedures in the United States [15]. This figure encompasses emergent as well as planned procedures. Neurosurgical operations have the potential to cause unavoidable damage to healthy brain tissue through application of pressure, tissue stretching, hemorrhage, and the use of electrocautery [16]. This form of brain injury occurs regularly, despite of intraoperative adjunct treatments such as administration of steroids and mannitol in both emergent or scheduled procedures [17]. Even standard microsurgical techniques have the ability to cause damage to the surrounding brain tissue, thus leading to early complications such as edema formation and local ischemia.

To determine the effects of hydrogen treatment following surgically-induced brain injury (SBI), Eckermann et al. utilized a novel rat model that involved a partial right frontal lobectomy [18]. The following groups were compared: rats subjected to sham operation (craniotomy), SBI animals that received $2.9 \%$ hydrogen, which was administered concurrently with surgery for a period of 0.5 hours, and a control group (SBI + room air). Brain water content and neurological deficits were measured at 24 hours after SBI. The results demonstrated that hydrogen treatment significantly decreased the formation of cerebral edema, which resulted in improved neurobehavioral function; however, the treatment failed to reduce oxidative stress and cerebral inflammation (evaluated via Lipid Peroxidase and Myeloperoxidase assays, respectively) in rats subjected to SBI.

\section{Stroke}

Stroke is a leading cause of death and long-term disability, particularly in the elderly population [19]. It is associated with a 30 -day mortality rate of approximately $20 \%$ [19]. The prevalence of stroke is expected to increase significantly as the global population of men and women - older than 65 years of age - increases continuously by an estimated 9 million people per year [20]. Consequently, it is most essential to investigate novel and potentially effective therapeutics to improve the neurofunctional outcome in patients. The effects of hydrogen treatment for ischemic stroke have been evaluated in an experimental rodent model of transient middle cerebral artery occlusion (tMCAO) [21]. This model exerts its damaging effect through focal ischemia and reperfusion, which generates acute oxidative stress to the affected brain regions. Under general anesthesia the rat's left middle cerebral artery (MCA) was occluded using a nylon monofilament with a distal silicon rubber tip. The treatment group inhaled $2 \%$ hydrogen gas during the entire procedure. Treated and control animals underwent neurological testing, and were sacrificed at 12 hours, 24 hours, 3 days, and 7 days post-surgery. Brains were then sectioned and stained with 2,3,5-triphenyltetrazolium chloride (TTC) to label the infarcted brain area, followed by volumetric computation of the infarct volume. Observed protective effects of hydrogen therapy included: decreased infarct volume, maintenance of body weight after surgery, and improved neurological function when compared to control animals. Liu et al. [22] utilized the tMCAO model to evaluate neuroprotective effects of intraperitoneally administered hydrogen saline ( $1 \mathrm{ml} / 100 \mathrm{~g}$ body weight). The results showed that hydrogen saline significantly reduced infarct volume, brain edema, and neurological function when administered within a 6 hour time window after ischemia induction. Hydrogen saline reduced ROS, inflammation markers, as well as caspase 3 activity in the ischemic brain [22]. Matchett et al. administered hydrogen gas $(2.9 \%)$ to adult rats that were subjected to tMCAO [23]. Hydrogen administration showed a tendency to reduce the infarction volume in the treatment group, when compared to control animals; however, neurological deficits were similar in both groups. Furthermore, Chen et al. [24] reported that hydrogen gas effectively reduced acute hyperglycemia-enhanced hemorrhagic transformation after focal ischemia in the rat. Potential mechanisms on how hydrogen gas ameliorates hemorrhagic transformation remain to be investigated.

Oshawa et al. demonstrated that hydrogen selectively reduced ROS in neuronal tissue cultures [21]. The effects were demonstrated via electron spin resonance signals in cells subjected to oxygen glucose deprivation followed by oxygen glucose reperfusion, which models cerebral ischemia-reperfusion in vitro [21]. After reperfusion, fluoroscopy demonstrated an immediate decrease in hydroxyl radicals $\left(\mathrm{OH}^{-}\right)$, and an increase in neuronal survival at 24 hours, suggesting that hydrogen effectively protects neurons from oxidative stress-mediated cell death.

Hemorrhagic stroke is often more severe than ischemic stroke, and includes intracerebral hemorrhages (ICH) and subarachnoid hemorrhages (SAH). The mortality rates for $\mathrm{ICH}$ and SAH have been reported as $40 \%$ to $50 \%$, and survivors are commonly affected by chronic morbidity [25]. Furthermore, the aggregate lifetime cost of hemorrhagic stroke cases has been estimated as high as $\$ 5.6$ to $\$ 6.0$ billion per year [26]. The effects of hydrogen administration in hemorrhagic stroke models have been previously reported. In a mouse model that implemented collagenase-induced $\mathrm{ICH}$, hydrogen therapy was found to significantly reduce cerebral edema 
and neurological deficits at 24 hours after surgery; however, hydrogen post-treatment showed only a tendency to improve these outcomes at 72 hours after surgery [27]. The authors conclude that hydrogen inhalation exerts an acute neuroprotective effect in mice subjected to experimental ICH. Similarly to these results, hydrogen treatment ( $2.9 \%$ for 2 hours) starting 1 hour after induction of experimental SAH (endovascular perforation method), ameliorated brain edema, reduced apoptosis, and improved neurological deficits at 24 hours, but not at 72 hours after surgery [28]. The protective effects of hydrogen therapy were associated with the reduction of oxidative injury of lipids, proteins, and DNA.

\section{Neonatal hypoxia-ischemia}

Advances in perinatal care and neonatology led to increased survival of premature infants [29], yet the incidence of brain injury caused by neonatal hypoxia-ischemia (HI) remains high [30]. Effective therapeutic modalities are limited for the treatment of HI. This form of CNS injury occurs at a very early stage in life. It is accompanied by a heavy toll on families and significant long-term healthcare demands. Since neonates have an immature free radical buffering system, they are believed to be more vulnerable to ROS damage.

Hydrogen treatment was associated with improved outcomes in a model of neonatal hypoxia/ischemia [31,32]. Cai et al. [32] subjected seven-day old rat pups to left common carotid artery ligation followed by 90 minutes of hypoxia. Immediately after the $\mathrm{HI}$ injury the pups were placed in chambers containing $2 \%$ hydrogen for 30, 60, and 120 minutes. Twenty-four hours following hydrogen therapy, the animals were sacrificed and the brain damage was assessed with TTC and Nissl staining, TUNEL, and evaluation of caspase activity. Hydrogen therapy significantly reduced the number of TUNEL positive cells, and attenuated caspase activity, suggesting that hydrogen gas exerts neuroprotective effects by inhibiting cellular apoptosis after HI. However, in another study of HI, hydrogen treatment failed to show neuroprotective effects, possibly as a result of the severity of the injury [23]. The authors concluded that hydrogen gas may have a neuroprotective effect against mild to moderate $\mathrm{HI}$, but loses that effect in cases of severe $\mathrm{HI}$ injury.

\section{Discussion}

Despite significant efforts directed towards the development of therapies that effectively reduce brain damage, particularly in TBI, only moderate success has been reported. So far, most advances involved preventative behavior modifications, such as wearing seat belts and helmets when using motor vehicles and motorcycles $[12,13]$. Further efforts need to be directed towards the search of neuroprotective agents that effectively improve the outcome of patients, even when applied post-injury. Many approaches have been suggested as post-treatments for CNS injuries such as hypothermia, vitamin E, hyperbaric oxygen, and numerous medications for ischemic and hemorrhagic strokes [33,34]. Post-injury protection reducing subsequent progression of the brain damage is critical; however, current success has been limited at best. Continued efforts are warranted to identify suitable neuroprotective agents that are inexpensive, easy to administer, and that present high therapeutic indexes. Increasing evidence suggests that hydrogen may be such an agent (Table 1). Several studies claim that hydrogen evokes cellular protection through attenuation of ROS production following diverse brain injuries. It has been demonstrated that hydrogen exerts a therapeutic antioxidant effect by selectively reducing hydroxyl radicals $\left(\mathrm{OH}^{-}\right)$, which are believed to be potent cytotoxic ROS, indiscriminately damaging proteins, lipids, and nucleic acids [21]. Furthermore, no known cellular detoxification system exists for $\mathrm{OH}^{-}$. Hydrogen also effectively protects cellular damage to non-neuronal tissues including renal, lung, and myocardial injury [21].

A recent clinical study by Ono et al. [35] showed that acute treatment with $\mathrm{OH}^{-}$scavengers (Edaravone and hydrogen) improved MRI indices (accelerated normalization) in patients with brainstem infarction. In fact, intravenous injection of Edaravone combined with hydrogen saline showed significantly better results than administration of Edaravone alone. The effects of hydrogen have also been examined in a randomized, doubleblinded, placebo-controlled crossover study implemented in patients with diabetes mellitus type 2 [36]. Patients that received $900 \mathrm{ml} /$ day of hydrogen-rich water for 8 weeks showed significant improvements of LDL cholesterol and urinary 8-isoprostanes. Further protective effects of hydrogen therapy were found in patients with mitochondrial myopathies, polymyositis and dermatomyositis [37].

Importantly, it needs to be determined if hydrogen treatment alters normal metabolism, since several agents classified as ROS, such as nitric oxide (NO), have important cell signaling functions and are regulators of physiologic responses [21]. It is crucial that the administered antioxidant does not disturb these normal cell-signaling pathways. Hydrogen may also function by preventing break-down of the $\mathrm{BBB}$. The most prominent and consistent effect is the reduction of cerebral edema and the improvement of neurological function postinjury. Hydrogen has several advantages as a potential antioxidant: it effectively reduces cytotoxic $\mathrm{OH}^{-}$in vivo, and demonstrates excellent penetration characteristics. Its ability to enter biomembranes and diffuse into mitochondria and cell nuclei render it highly effective as a powerful antioxidant [21]. 
Table 1 Animal studies examining neuroprotection by hydrogen

\begin{tabular}{|c|c|c|c|c|c|}
\hline Author & Year & Model & Species & Effects of $\mathrm{H}_{2}$ & Proposed Mechanism \\
\hline Ji et al. [14] & 2010 & $\mathrm{~TB} \mid$ & Rat & Brain edema $\downarrow$, neurological deficits $\downarrow$ & $\begin{array}{c}\mathrm{H}_{2} \text { increased endogenous antioxidant enzymatic } \\
\text { activities }\end{array}$ \\
\hline $\begin{array}{l}\text { Eckermann et al. } \\
{[18]}\end{array}$ & 2011 & $\mathrm{SBl}$ & Rat & Brain edema $\downarrow$, neurological deficits $\downarrow$ & None proposed \\
\hline Ohsawa et al [21] & 2007 & tMCAO & Rat & Brain infarction $\downarrow$, neurological deficits $\downarrow$ & $\mathrm{H}_{2}$ selectively inhibited $\mathrm{OH}^{-}$ \\
\hline $\begin{array}{l}\text { Matchett et al. } \\
\text { [23] }\end{array}$ & 2009 & tMCAO & Rat & Tendentially reduced brain infarction & None proposed \\
\hline Chen et al. [24] & 2010 & tMCAO & $\begin{array}{l}\text { Hyperglycemic } \\
\text { rat }\end{array}$ & Hemorrhagic transformation $\downarrow$ & $\mathrm{H}_{2}$ reduced hyperglycemia \\
\hline Liu et al. [22] & 2011 & tMCAO & Rat & $\begin{array}{c}\text { Brain edema } \downarrow \text {, infarction } \downarrow \text {, neurological } \\
\text { deficits } \downarrow\end{array}$ & ROS $\downarrow$, inflammation $\downarrow$, apoptosis $\downarrow$ \\
\hline $\begin{array}{l}\text { Manaenko et al. } \\
{[27]}\end{array}$ & 2011 & $\mathrm{ICH}$ & Mouse & $\begin{array}{c}\text { Acute brain edema } \downarrow \text {, neurological } \\
\text { deficits } \downarrow\end{array}$ & None proposed \\
\hline Zhan et al. [28] & 2012 & $\mathrm{SAH}$ & Rat & $\begin{array}{c}\text { Acute brain edema } \downarrow \text {, neurological } \\
\text { deficits } \downarrow\end{array}$ & BBB permeability $\downarrow$, ROS $\downarrow$, apoptosis $\downarrow$ \\
\hline Matchet et al. [23] & 2009 & $\mathrm{HI}$ & Mouse & No beneficial effects & None proposed \\
\hline Cai et al. [31] & 2008 & $\mathrm{HI}$ & Rat & Brain infarction $\downarrow$, apoptosis $\downarrow$ & $\mathrm{H}_{2}$ selectively inhibited caspase activity \\
\hline
\end{tabular}

Utilized models include traumatic brain injury (TBI), surgically induced brain injury (SBI), transient middle cerebral artery occlusion (tMCAO), intracerebral hemorrhage $(\mathrm{ICH})$, subarachnoid hemorrhage $(\mathrm{SAH})$ and neonatal hypoxia-ischemia $(\mathrm{HI})$

Taken together, hydrogen appears to be a safe therapeutic with the ability to attenuate ROS production after diverse CNS injuries. Furthermore, hydrogen effectively reduced neuronal apoptosis and hemorrhagic transformation after ischemic brain injury. Its ease of use and availability will facilitate clinical transition in the near future; however, further mechanisms of neuroprotection need to be primarily investigated.

\section{Abbreviations}

BBB: Blood brain barrier; $\mathrm{H}_{2}$ : Hydrogen; $\mathrm{H}_{2} \mathrm{~S}$ : Hydrogen sulfide; $\mathrm{HI}$ : Neonatal hypoxia-ischemia; ICH: Intracerebral hemorrhage; ROS: Reactive oxygen species; MCA: Middle cerebral artery; SAH: Subarachnoid hemorrhage; TBI: Traumatic brain injury; tMCAO: Transient middle cerebral artery occlusion; TTC: 2:3:5-triphenyltetrazolium chloride.

\section{Author details \\ 'Department of Neurosurgery, Stanford University School of Medicine, Stanford, California, USA. ²Department of Radiation Oncology, Stanford University School of Medicine, Stanford, California, USA. ${ }^{3}$ Department of Physiology, Loma Linda University School of Medicine, Loma Linda, California, USA. ${ }^{4}$ Department of Neurosurgery, Loma Linda University School of Medicine, Loma Linda, California, USA. ${ }^{5}$ Department of Neurosurgery, Stanford Hospital and Clinics, 300 Pasteur Drive, Room R281, Stanford, CA 94304, USA}

\section{Authors' contributions}

JE initiated this study. He conducted the literature review together with PK LS, RL and SD. JE and PK provided review of the use of hydrogen in traumatic and surgically induced brain injury. LS, RL focused on ischemic and hemorrhagic stroke. SD provided review of the use of hydrogen in neonatal hypoxia-ischemia. All authors discussed their individual findings with all co-authors and collectively developed the discussion section of this paper. AC supervised all meetings for discussion and made substantial contributions to the overall content. AC also edited this paper. All authors have read and approved the final manuscript.

\section{Competing interests}

The authors declare that they have no competing interests.
Received: 3 January 2012 Accepted: 19 April 2012

Published: 19 April 2012

\section{References}

1. Tan BH, Wong PT, Bian JS: Hydrogen sulfide: a novel signaling molecule in the central nervous system. Neurochem Int 2010, 56(1):3-10.

2. Qu K, et al: Hydrogen sulfide is a mediator of cerebral ischemic damage. Stroke 2006, 37(3):889-893.

3. Lowicka $\mathrm{E}$, Beltowski J: Hydrogen sulfide $(\mathrm{H} 2 \mathrm{~S})$ - the third gas of interest for pharmacologists. Pharmacol Rep 2007, 59(1):4-24.

4. Kimura H: Hydrogen sulfide as a neuromodulator. Mol Neurobiol 2002, 26(1):13-19.

5. Guidotti TL: Hydrogen sulfide: advances in understanding human toxicity. Int J Toxicol 2010, 29(6):569-581.

6. Szabo C: Gaseotransmitters: new frontiers for translational science. Sci Transl Med 2010, 2(59):p. 59-ps54.

7. Huang CS, et al: Recent advances in hydrogen research as a therapeutic medical gas. Free Radic Res 2010, 44(9):971-982.

8. Yang $Z$, et al: Extrinsic Fabry-Perot interferometric optical fiber hydrogen detection system. Appl Opt 2010, 49(15):2736-2740.

9. Gardette B, Delauze HG: Techniques of underwater intervention: means, methods, research and outlook. Bull Acad Natl Med 1996, 180(5):975-983.

10. Dutton RP, et al: Trauma mortality in mature trauma systems: are we doing better? An analysis of trauma mortality patterns, 1997-2008. J Trauma 2010, 69(3):620-626.

11. Thurman DJ, et al: Traumatic brain injury in the United States: A public health perspective. J Head Trauma Rehabil 1999, 14(6):602-615.

12. Langlois JA, Rutland-Brown W, Wald MM: The epidemiology and impact of traumatic brain injury: a brief overview. J Head Trauma Rehabil 2006, 21(5):375-378.

13. Langlois JA, et al: Traumatic brain injury-related hospital discharges. Results from a 14-state surveillance system, 1997. MMWR Surveill Summ 2003, 52(4):1-20

14. Ji $X$, et al: Beneficial effects of hydrogen gas in a rat model of traumatic brain injury via reducing oxidative stress. Brain Res 2010, 1354:196-205.

15. Etzioni DA, et al: The aging population and its impact on the surgery workforce. Ann Surg 2003, 238(2):170-177.

16. Elliott-Lewis EW, Mason AM, Barrow DL: Evaluation of a new bipolar coagulation forceps in a thermal damage assessment. Neurosurgery 2009, 65(6):1182-1187, discussion 1187.

17. Jadhav $\mathrm{V}$, Zhang $\mathrm{JH}$ : Surgical brain injury: prevention is better than cure. Front Biosci 2008, 13:3793-3797.

18. Eckermann $\mathrm{JM}$, et al: Hydrogen is neuroprotective against surgically induced brain injury. Med Gas Res 2011, 1(1):7. 
19. Tegos TJ, et al: Stroke: epidemiology, clinical picture, and risk factors-Part I of III. Angiology 2000, 51(10):793-808.

20. Mukherjee D, Patil CG: Epidemiology and the global burden of stroke. World Neurosurg 2011, 76(6 Suppl):S85-S90.

21. Ohsawa I, et al: Hydrogen acts as a therapeutic antioxidant by selectively reducing cytotoxic oxygen radicals. Nat Med 2007, 13(6):688-694.

22. Liu $Y$, et al: Hydrogen saline offers neuroprotection by reducing oxidative stress in a focal cerebral ischemia-reperfusion rat model. Med Gas Res 2011, 1(1):15.

23. Matchett $G A$, et al: Hydrogen gas is ineffective in moderate and severe neonatal hypoxia-ischemia rat models. Brain Res 2009, 1259:90-97.

24. Chen $\mathrm{CH}$, et al: Hydrogen gas reduced acute hyperglycemia-enhanced hemorrhagic transformation in a focal ischemia rat model. Neuroscience 2010, 169(1):402-414

25. Woo $D$, et al: Effect of untreated hypertension on hemorrhagic stroke. Stroke 2004, 35(7):1703-1708.

26. Taylor TN, et al: Lifetime cost of stroke in the United States. Stroke 1996, 27(9):1459-1466

27. Manaenko A, et al: Hydrogen inhalation is neuroprotective and improves functional outcomes in mice after intracerebral hemorrhage. Acta neurochirurgica Supplement 2011, 111:179-183.

28. Zhan $Y$, et al: Hydrogen gas ameliorates oxidative stress in early brain injury after subarachnoid hemorrhage in rats. Crit Care Med 2012, 40(4):1291-1296

29. Smith $L K$, et al: Nature of socioeconomic inequalities in neonatal mortality: population based study. BMJ 2010, 341:c6654.

30. Lawrence RK, Inder TE: Anatomic changes and imaging in assessing brain injury in the term infant. Clin Perinatol 2008, 35(4):679-693.

31. Cai J, et al: Hydrogen therapy reduces apoptosis in neonatal hypoxiaischemia rat model. Neurosci Lett 2008, 441(2):167-172.

32. Cai J, et al: Neuroprotective effects of hydrogen saline in neonatal hypoxia-ischemia rat model. Brain Res 2009, 1256:129-137.

33. Laskowitz DT, Kolls BJ: Neuroprotection in subarachnoid hemorrhage. Stroke 2010, 41(10 Suppl):S79-S84.

34. Kellner CP, Connolly ES Jr: Neuroprotective strategies for intracerebral hemorrhage: trials and translation. Stroke 2010, 41(10 Suppl):S99-S102.

35. Ono $\mathrm{H}$, et al: Improved brain MRI indices in the acute brain stem infarct sites treated with hydroxyl radical scavengers, Edaravone and hydrogen as compared to Edaravone alone. A non-controlled study. Med Gas Res 2011, 1(1):12.

36. Kajiyama S, et al: Supplementation of hydrogen-rich water improves lipid and glucose metabolism in patients with type 2 diabetes or impaired glucose tolerance. Nutr Res 2008, 28(3):137-143.

37. Ito $\mathrm{M}$, et al: Open-label trial and randomized, double-blind, placebocontrolled, crossover trial of hydrogen-enriched water for mitochondrial and inflammatory myopathies. Med Gas Res 2011, 1(1):24.

doi:10.1186/2045-9912-2-11

Cite this article as: Eckermann et al:: Potential application of hydrogen in traumatic and surgical brain injury, stroke and neonatal hypoxiaischemia. Medical Gas Research 2012 2:11.

\section{Submit your next manuscript to BioMed Central and take full advantage of:}

- Convenient online submission

- Thorough peer review

- No space constraints or color figure charges

- Immediate publication on acceptance

- Inclusion in PubMed, CAS, Scopus and Google Scholar

- Research which is freely available for redistribution

Submit your manuscript at www.biomedcentral.com/submit
Biomed Central 